\title{
Randomness and metastability in CDMA paradigms
}

\author{
Jack Raymond, David Saad \\ Aston University, Neural Computing Research Group, Birmingham, B4 7ET, UK \\ Email: raymonjr@aston.ac.uk
}

\begin{abstract}
Code Division Multiple Access (CDMA) in which the signature code assignment to users contains a random element has recently become a cornerstone of CDMA research. The random element in the construction is particularly attractive in that it provides robustness and flexibility in application, whilst not making significant sacrifices in terms of multiuser efficiency. We present results for sparse random codes of two types, with and without modulation. Simple microscopic consideration on system samples would suggest large differences in the phase space of the two models, but we demonstrate that the thermodynamic results and metastable states are equivalent. This may have consequences for developing algorithmic methods to escape metastable states, thus improving decoding performance.
\end{abstract}

\section{INTRODUCTION}

The area of multiuser communications is one of great interest from both theoretical and engineering perspectives [Ver98]. Code Division Multiple Access (CDMA) is a particular method for allowing multiple users to access channel resources in an efficient and robust manner, and plays an important role in the current standards for allocating channel resources in wireless communications. CDMA utilises channel resources highly efficiently by allowing many users to transmit on much of the bandwidth simultaneously, each transmission being encoded with a user specific signature code. Disentangling the information in the channel is possible by using the properties of these codes and much of the focus in CDMA research is on developing efficient codes and decoding methods.

A typical CDMA paradigm is that bandwidth is broken into $N$ discrete Time-Frequency blocks (chips) with each of $K$ users being assigned a user code $\left(\vec{s}_{k}\right)$ known by the base station, the set of all user codes being $s$ (the code). The user code gives the amplitude and phase by which to modulate transmission of the scalar symbol on each chip. The signal $(\vec{y})$ received on $N$ chips by the base station is then an interfering (additive) combination of the users' modulated symbols corrupted during transmission by a fading factor $F_{k \mu}$ and some signal noise $\left(\nu_{\mu}\right)$. Assuming perfect synchronisation of the chips the symbols received on each chip are independent and given by

$$
y_{\mu}=\nu_{\mu}+\sum_{k=1}^{K} b_{k} F_{k \mu} s_{k \mu} .
$$

We focus on a standard channel type (BIAWGN): the Additive White Gaussian Noise channel (AWGN), employing Binary Phase Shift Keying (BPSK). The following parameterisations are assumed: the scalar symbol sent by user $k$ is a bit $b_{k}= \pm 1$ with probability $P_{b_{k}}(b)=\frac{1}{2}$; the noise is Gaussian with zero mean and variance $\sigma_{0}^{2}$ for all chips; prefect power control applies so that the fading factor $F_{k \mu}=1$; each code element $s_{\mu k}= \pm A$, where $A$ is the amplitude of the transmission by user $k$ on chip $\mu$. Generalisations of the model most often consider the requirement for perfect synchronisation and power control. Real CDMA applications also have to deal with idiosynchracies in hardware and environmental conditions not easy to treat in a generalised analysis, this has not prevented its updake in some modern wireless communication standards.

This paper follows previous theoretical analyses (e.g. [Tan02], [YT06], [MPT06], [RS07]) in studying codes which are randomly generated for each system from some ensemble. The canonical random CDMA ensemble is the dense one in which all chips are transmitted upon [Ver98]. In the sparse ensemble we consider here (2) only a small number of chips $O(C)$ are accessed by each user, a less studied system. However there are a number of reasons why the sparse ensemble first examined in [YT06] may be more practical, based on its closer similarity to FH/TH-CDMA and the ability to apply fast message passing algorithms in decoding. In addition, one can converge towards the properties of the dense ensemble by increasing the mean user connectivity $C$ only moderately. It has been shown, for a sparse connectivity model in which the mean user connectivity is large but much smaller than $K$, that the properties become indistinguishable from the dense channel in cases where BP converges [GW07].

The sparse codes consist of a sparse connectivity matrix and a modulation part sampled according to

$$
\begin{aligned}
P_{\mathbf{s}}(\mathbf{x}) & \propto \prod_{k} \prod_{\mu}\left[\left(1-\frac{L}{K}\right) \delta_{x_{\mu k}}+\frac{L}{K} \phi\left(x_{\mu k}\right)\right] \\
\phi(x) & =\frac{1}{2}\left(\delta_{x, A}+\delta_{x,-A}\right) .
\end{aligned}
$$

The modulation of non-zero elements in the codes is described by $\phi$ which can be BPSK (as shown) or unmodulated $\phi(x)=$ $\delta_{x, A}$, with the amplitude of transmission $(A=1 / \sqrt{L})$ chosen for normalisation purposes so that the Power Spectral density $\mathcal{Q}$, a representative measure of signal to noise ratio, may be taken as $1 /\left(2 \sigma_{0}^{2}\right)$. The mean chip and user connectivities are $L$ and $C$, respectively, such that the load $\alpha=L / C=K / N$.

Two problems with the basic sparse ensemble (2) at low connectivity is significant asymmetry in bandwidth access for users, with a fraction of users being entirely disconnected. Analogously the utilisation of chips will not be uniform, with some chips unutilised. These problems can be overcome by 
enforcing regularity of the following forms:

$$
\begin{aligned}
P_{\mathbf{s}}(\mathbf{x}) & \propto \prod_{k}\left[\delta\left(\sum_{\mu}^{N}\left(1-\delta_{x_{\mu k}}\right)-C\right)\right], \\
& \propto \prod_{k}[. .] \prod_{\mu}\left[\delta\left(\sum_{k}^{K}\left(1-\delta_{x_{\mu k}}\right)-L\right)\right],
\end{aligned}
$$

in addition to modulation though $\phi$. It turns out that constraining users to access exactly $C$ chips (4) is very important in attaining near optimal performance for high $\mathcal{Q}$, whereas enforcing, in addition, chip regular access (5) produces only marginally improved performance [RS07] and may be difficult to implement in practice. In this paper we consider ensembles with both chip and user regular constraints (5) throughout since it makes certain aspects of the analysis simpler; we anticipate results to be qualitatively similar with only the userregular constraint (4).

The theoretical information capacity, and theory of Bayes optimal decoding requires knowledge of the likelihood of transmitted bits

$$
P_{\vec{y} \mid \vec{b}}(\vec{\tau}) \propto \int \prod_{\mu}\left[\delta\left(y_{\mu}-\sum_{k} s_{\mu k} \tau_{k}+\omega_{\mu}\right)\right] \hat{P}_{\vec{\nu}}(\vec{\omega}) d \vec{\omega}
$$

where $\hat{P}_{\vec{\nu}}$ is the assumed chip noise distribution to be marginalised over. If one considers a Gaussian channel noise model, of variance $\left(\sigma_{0}\right)^{2} / \beta$ (i.e assumption possibly incorrect by a factor $\beta$ ), then the righthand side is simplified

$$
P_{\vec{b} \mid \vec{y}}(\vec{\tau}) \propto \prod_{\mu} \exp \left\{-\beta \mathcal{Q}\left(y_{\mu}-\sum_{k} s_{\mu k} \tau_{k}\right)^{2}\right\} .
$$

Statistical physics provides a concise framework to analyse this quantity. First we define a Hamiltonian by connection with the likelihood

$$
\mathcal{H}(\vec{\tau})=-\mathcal{Q} \sum_{\mu}\left(\nu_{\mu}+\sum_{k} s_{\mu k}\left(b_{k}-\tau_{k}\right)\right)^{2},
$$

where $y_{\mu}$ is written in terms of its constituent components (1) and $\tau_{k}$ is a candidate value of the sent bit. From this one can construct the self-averaging free energy.

$$
f=\left\langle-\frac{1}{\beta N} \log \sum_{\vec{\tau}} \exp \{-\beta \mathcal{H}(\vec{\tau})\}\right\rangle .
$$

The average \langle\rangle denotes throughout the paper an average over $\vec{y}$ and codes s sampled according to the appropriate ensemble. The motivation for studying the self-averaged free energy that this is a generating function for many interesting statistics attainable by decoders, averaged over samples of the system. It can be observed that for CDMA the performance measures, such as bit error rate and spectral efficiency, are self-averaging - rapidly converging to some fixed values as the number of users increase. The bit error rate is mean overlap of the sent and decoded bits $\frac{1}{K}\langle(\vec{b} \cdot \vec{\tau})\rangle$, the spectral efficiency is the mutual information between the sent bits and the received

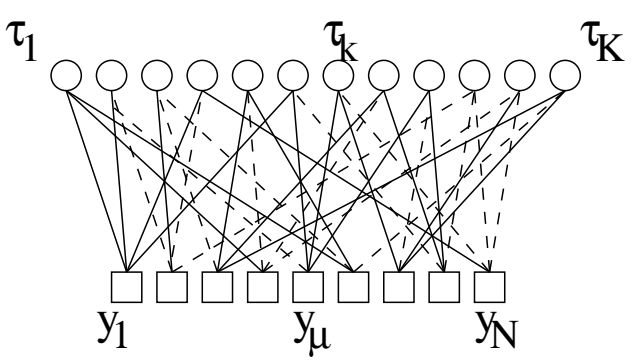

Fig. 1. The inference problem can be represented by a graphical model: a Tanner (or factor) graph. Each factor (square) represents an interaction and each bit (circle) denotes a dynamical variable $\tau_{k}$ which is to be optimised given the topology and observable values. The observables in this case are the signal $y_{\mu}$ associated to each node, and the code $\mathbf{s}-($ dashed/solid lines can be used to indicate modulation by $\pm A$ in components $s_{\mu k}$ ). Above is a representation for a small sparse regular graph $(5,4)$ with $L=4 C=3$.

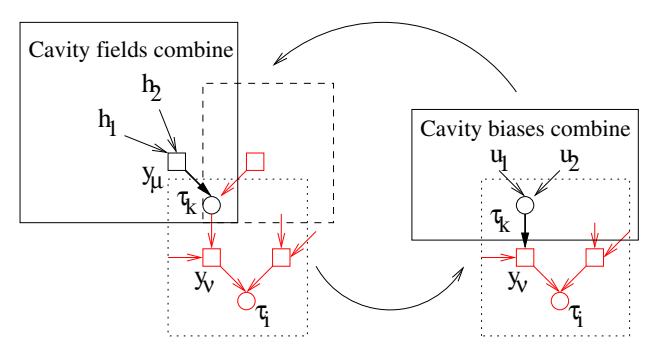

Fig. 2. The fixed points of the self consistent equations are in quantities $h$ and $u$ which have an interpretation in terms of messages passed on (sub)graphs of the graphical model (1). If one knows the log likelihood ratio $u_{\mu k}$ of bit $b_{k}$ given only one of its neighbours $\mu$, then assuming these likelihoods to be independent (as is valid on a tree), one can construct the conditional likelihood of $b_{k}$ given all its neighbours excluding $\nu$ (or log likelihood ratio $\left.h_{k \nu}\right)$. One can then use $h_{k \nu}$ to construct log likelihoods $\left(u_{\nu i}\right)$ for subsequent variables in the tree. By such a process, the distribution of $\{h\}$ and $\{u\}$ may converge at sufficient depth in the tree to values independent of the inputs such a solution is a viable solution to a population dynamics algorithm. The convergence properties and stability of solutions is closely related to standard decoding algorithms: the sum product algorithm or belief propagation.

signal $I(\vec{b}, \vec{y})$ and is affine to the free energy. By taking the limit $K \rightarrow \infty$ we are able to attain an exact description for these fixed points, thereby providing a good indication of performance. We assume throughout this proceedings that $\beta=1$, analysis of the free energy thereby corresponds to the performance of a detector which minimises the bit error rate.

\section{A. Overview of results for BPSK}

For sparse ensembles with BPSK the equilibrium and dynamical properties are similar to the dense case [Tan02], becoming more so as $L$ increases [GW07]. If one calculates the free energy of the sparse ensemble by the cavity or replica method [MPV87] one attains under assumptions of a single pure state a site factorised expression for the free energy, determined by the solution to a set of self consistent field and bias distributions (saddlepoint equations) [RS07]. These 


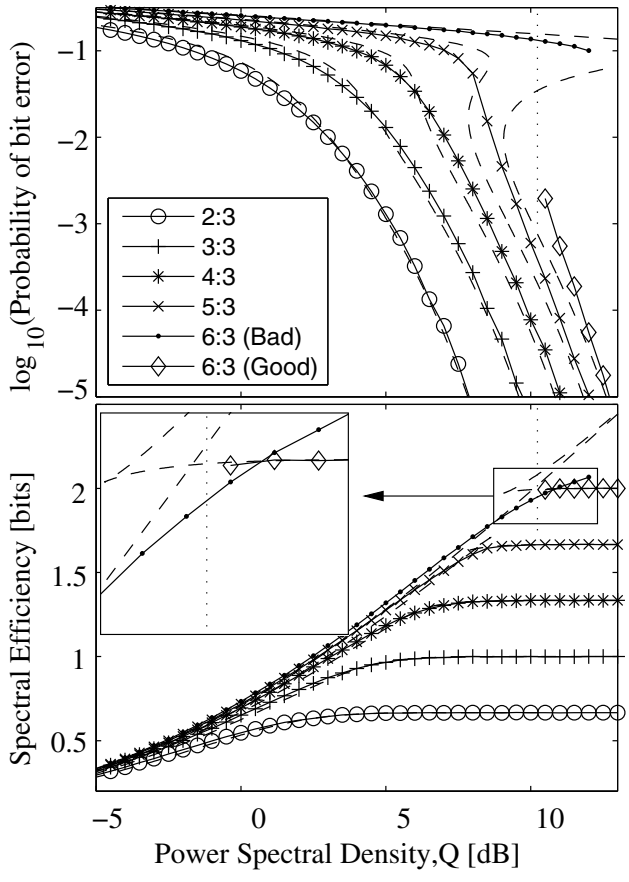

Fig. 3. The figures show the spectral efficiency (affine to the free energy) and bit error rate for a number of cases of $\alpha$ as indicated by $K: N$. The solid curves represent locally stable solutions of the population dynamics procedure for a sparse ensemble, dashed curves show the exact results for the $\mathcal{Q}$-equivalent densely spread CDMA system - the curves are qualitatively similar in both quantities, except in the existence of one additional (unstable) solution in the dense case (middle curve). The similarity extends to the metastable ranges, we consider the sparse ensemble results in detail. The sparse ensemble is fully regular with $C=3$ and $L=2, \ldots, 6$ in agreement with the ratio $\alpha$. For small loads $\alpha$ a unique solution is found in both cases, which is the valid thermodynamic (information theoretic) solution. For the sparse case at sufficiently large $\alpha$ (case 6:3) the solution becomes multivalued. Lower figure: The thermodynamic solution is the curve of lowest spectral efficiency, the other being metastable; there is a second order transition between the two solution with increasing $\mathcal{Q}$. The inset shows in detail the region in which the dense and sparse codes undergo thermodynamic second order transitions with $\alpha=2$. Upper figure: This demonstrates the bit error rate for comparable parameterisations. This figure indicates a large performance gap between the two locally stable solutions in the metastable regime: a bad and good solution exist in terms of decoding. The vertical dashed line indicates the smallest $\mathcal{Q}$ at which metastability occurs in the sparse code for the 6:3 case: beyond this point in the metastable regime the bad solution performance is typically attained by belief propagation even if this is only a metastable solution.

results are presented for later comparison (12)

$$
\begin{aligned}
W(h) & \propto \int \prod_{c=1}^{C-1}\left[d u_{c} \hat{W}\left(u_{c}\right)\right] \delta\left(h-\sum_{c=1}^{C-1} u_{c}\right) \\
\hat{W}(u) & \propto \int \prod_{l=1}^{L-1}\left[W\left(h_{l}\right) d h_{l}\right] \prod_{l=1}^{L}\left[\phi\left(x_{l}\right) d x_{l}\right] P_{\nu}(\omega) d \omega \\
& \times \delta\left(u-\sum_{\tau_{L}} \tau_{L} \log \left(\mathcal{Z}\left(\tau_{L}\right)\right)\right) \\
\mathcal{Z}\left(\tau_{L}\right) & =\sum_{\vec{\tau}} \exp \left\{-\mathcal{Q}\left(\omega+\sum_{l=1}^{L} x_{l}\left(1-\tau_{l}\right)\right)^{2}+\sum_{l} h_{l} \tau_{l}\right\}
\end{aligned}
$$

where $P_{\nu}$ is the true chip noise probability distribution. The distributions are over a set of cavity biases $u$ and cavity fields $h$. These variables may be interpreted within a graphical framework of the inference problem (Fig. 1), as log-likelihood (of correct decoding) ratios in two types of sub-graphs (Fig. 2). From these distributions one can calculate the free energy, bit error rate and other properties. The equations may be solved numerically by population dynamics [RS07], which is implemented as a late propagation (decoding) algorithm on a tree. This processes allows a numerical determination of the free energy and tests of ergodicity breaking. We find a unique thermodynamic solution at all $\mathcal{Q}$, but also a significant metastable solution for a range of parameters (Fig. 3).

We may distinguish the metastable states in this range of parameters as bad and good (higher or lower bit error rate). The population dynamics algorithm tends to find the bad solution from most initial conditions, only those initial conditions which are of very low bit error rate (a set of cavity biases strongly correlated with $\vec{b}$ ) appear to converge towards the good solution. It appears the bad solution is easy to reach by implementation of population dynamics regardless of whether it is the thermodynamically dominant state. This is interesting since population dynamics appears to mirror the behaviour of many decoding algorithms on even relatively small systems, which struggle to achieve good bit error rates in this region. In the real decoding problem one does not begin the decoding already with a good estimate of $\vec{b}$, and so one may be stuck with a suboptimal estimate even where a much better estimate may be found (in principle) for almost all decodings.

In both the dense and sparse cases there is a unique thermodynamically stable state. One can hope to achieve the information capacity of the thermodynamic state by clever algorihms based on some global insight. The problem is that local search based optimisation appears insufficient. In the case of no metastability, local search methods attain the optimal solution [GW07], [RS07] with various principled modifications suggested [Kab03]. In the case of metastability one might apply a principle of guesswork combined with BP to allow efficient searching of the space. Such a method [MMU05] has been demonstrated for certain types of channel, unfortunately not so far the BIAWGN we consider. In the following sections we consider how the similarity between the phenomena in dense and sparse systems, combined with a consideration of marginal interaction distributions, might characterise the bad metastable solution and how such insight might be used to supplement local search methods.

\section{B. A sparse model without modulation}

As a way to further understand the microscopic basis of metastability we propose the following model to investigate the sparse ensemble for the case of no modulation, $\phi(x)=\delta_{x, A}$. Unlike the dense model, the disorder in the connectivity structure is sufficient to recover information even without modulation. Given that the graphical structure is identical to the modulated sparse ensemble, decoding may be achieved by similar methods (belief propagation based local search).

Working with either the cavity or replica methods one can attain a site factorised set of functional relations analogous to 
(10). In the former case we had two distributions containing information on the probabilty of correct bit reconstruction (on two types of subgraph). In the unmodulated case we replace each of these distributions by two, because the probability of correct bit recovery is dependent on the candidate bit at the given site, $\tau_{k}=a$. Assuming no ergodicity breaking one can attain the variational part of the free energy density ((9) in the large $N$ limit) as

$$
\begin{aligned}
f & =\sum_{a} \int d h d u W(a, h) \hat{W}(a, u) \log (1+\tanh (u) \tanh (h)) \\
& +\alpha \sum_{a} P_{b}(a)\left\{C \int d u W(a, u) \log (\cosh u)\right. \\
& \left.+\int \prod_{c=1}^{C}\left[d u_{c} W\left(a, u_{c}\right)\right] \log \left(\cosh \left(\sum_{c=1}^{C} u_{c}\right)\right)\right\} \quad(11) \\
& +\int \prod_{l=1}^{L}\left[d x_{l} d \phi\left(x_{l}\right) \sum_{a_{l}} d h_{l} W\left(a_{l}, h_{l}\right)\right] d \omega P_{\nu}(\omega) \log \mathcal{Z}_{I} \\
\mathcal{Z}_{I} & =\sum_{\vec{\tau}} \prod_{l=1}^{L}\left[\frac{\exp \left(h_{l} \tau_{l}\right)}{2 \cosh \left(h_{l}\right)}\right] \exp \left\{\mathcal{Q}\left(\omega+\sum_{l=1}^{L} x_{l} a_{l}\left(1-\tau_{l}\right)\right)^{2}\right\}
\end{aligned}
$$

Here $P_{b}$ is the true prior on transmitted bits, which we will assume to be uniform. We also assume the sparse ensemble with chip and user regularity for brevity. The distributions must be chosen to minimise the free energy, it is a near identical minimisation which gives rise to (10). The pairs of field and bias distributions $\hat{W}, W$, in this case obey the saddlepoint equations

$$
\begin{aligned}
W(a, h) & \propto \int \prod_{c=1}^{C-1}\left[d u_{c} \hat{W}\left(a, u_{c}\right)\right] \delta\left(h-\sum_{c=1}^{C-1} u_{c}\right) \\
\hat{W}\left(a_{L}, u\right) & \propto \int \prod_{l=1}^{L-1}\left[\phi\left(x_{l}\right) d x_{l} \sum_{a_{l}} W\left(a_{l}, h_{l}\right) d h_{l}\right] P_{\nu}(\omega) d \omega \\
& \times \delta\left(u-\sum_{\tau_{L}} \tau_{L} \log \left(\mathcal{Z}\left(\tau_{L}\right)\right)\right)
\end{aligned}
$$

Where $\mathcal{Z}$ is the same quantity as (10) upto the substitution of $x_{l}$ by $a_{l}$. In this new case we have a modified set of equations on distributions, as the dependence on the root site cannot be factorised. Since we are considering maximal rate both in the prior for sent message and inference model we can argue by symmetry that $W(b, h)$ equals $W(-b, h)$. This represents the intuitive statement that the probability of correct reconstruction is independent of whether the sent bit is \pm 1 , however this is an ansatz rather than a result of the calculation. The assumption can be tested by allowing convergence restricted to the symmetric combination and testing small perturbations in the antisymmetric part. A stronger test of the ansatz is to allow the population dynamics to run with fully independent distributions. To within numerical accuracy the restricted solutions and those found in this larger space appear to be consistent and the modulated and unmodulated sparse ensembles become equivalent. At maximal rate the solution for the unmodulated ensemble is information theoretically equivalent to the unmodulated ensemble.

\section{NATURE OF THE METASTABle SOlUtions}

The exact results and numerical solutions (as indicated by example in Fig. 3) indicate several features of the metastable state for both the sparse and dense systems. We investigate these points and present some simplified analysis of the energy landscape in this section. The results of the previous section provide insight into the probable nature of the state, and the fact that the sparse and dense systems are so similar qualitatively means that topology must play a relatively small role. The dynamical properties of the decoding algorithms reported for both cases appear to be an important common feature, while the sizes of solutions (as indicated by entropy) and bit error rates reduce the space of solutions to be considered.

One can gain further insight by examining the interaction structure as a source of information, making analogies between other well studied disordered systems [MPV87]. The Hamiltonian may be re-written (upto constants) as

$$
\mathcal{H}(\vec{\tau})=-\left(\sum_{k \neq k^{\prime}} J_{k k^{\prime}} \tau_{k} \tau_{k^{\prime}}+\sum_{k} h_{k} \tau_{k}\right)
$$

which is a standard formulation in physics, where the set of couplings $J_{i j}$ and fields $h_{i}$ describe the problem

$$
\begin{aligned}
J_{k, k^{\prime}} & =\mathcal{Q} \sum_{\mu} s_{\mu k} s_{\mu k^{\prime}} \\
h_{k} & =2 \mathcal{Q} \sum_{\mu} y_{\mu} s_{\mu k}=2 \mathcal{Q}\left[\sum_{\mu} s_{\mu k}^{2}\right] b_{k} \\
& \left.\left.+2 \mathcal{Q}\left(\left\{\sum_{\mu} s_{\mu k} \sum_{k^{\prime}(\neq k)} s_{\mu k^{\prime}} b_{k^{\prime}}\right\}\right\}+\left\{\sum_{\mu} \nu_{\mu} s_{\mu k}\right\}\right\}\right)
\end{aligned}
$$

Since the coupling term has no dependence on the sent bits $\vec{b}$ the states induced by the couplings alone must be uncorrelated with the true solution. By contrast, the field term encodes a bias towards the sent vector combined with a pair of fields with no alignment along the correct solution (in expectation), but with some dependence thereof.

The couplings and fields are strongly correlated through the code $\mathbf{s}$. In the case of a dense code where $L \rightarrow K$ both marginal distributions over couplings and fields may be taken as Gaussian distributed through application of the central limit theorem with $N=K / \alpha$ large; the dense case gives

$$
\begin{aligned}
P\left(J_{k, k^{\prime}}\right) & =\mathcal{N}\left(0, \frac{\mathcal{Q}^{2}}{\alpha N}\right), \\
P\left(h_{k}\right) & =\mathcal{N}\left(\frac{2 \mathcal{Q} b_{k}}{\alpha}, \frac{(2 \mathcal{Q})^{2}}{\alpha}+\frac{2 \mathcal{Q}}{\alpha}\right) .
\end{aligned}
$$

where $\mathcal{N}$ signifies the normal distribution. The first term of the field variance is negligable for the large system.

For the sparse code with BPSK one can instead note that the couplings are non-zero with probability $\left(\begin{array}{l}L \\ 2\end{array}\right) /\left(\begin{array}{l}K \\ L\end{array}\right)$ reflecting the enforced topology (2),(4),(5), and in the non-zero cases take 
values $\pm \mathcal{Q} / L$ with equal probability. In the field part one has a net positive field combined with two terms, the first term containing no noisy part gives a variance dependent on the site values and number of nearest neighbours (users connected through chips to user $k$ ), whereas the second is the sum of Gaussian random variables associated to each neighbouring chip. We approximate the distribution by a mean and variance to abbreviate this information, ignoring for convenience higher order moments as

$$
P\left(h_{k}\right)=\mathcal{N}\left(\frac{(2 \mathcal{Q}) b_{k}}{\alpha}, \frac{(L-1)(2 \mathcal{Q})^{2}}{\alpha L}+\frac{2 \mathcal{Q}}{\alpha}\right) .
$$

The $L-1$ prefactor is the average excess degree of the factor node in the chip regular ensemble (5), for the random graph ensemble (2) the value is $L$ (also with user regularity (4)). Using a non-regular code appears to impact upon the variance of the field but not the mean.

When one does not include the BPSK, the first two moments of the sparse distribution of local fields (17) are unchanged but the couplings are entirely ferromagnetic $+\mathcal{Q} / L$, again conforming to the underlying topology. At least for $\beta=1$ we have determined that the information theoretical quantities, and the population dynamics algorithm are equivalent for the two sparse ensembles considered. Therefore we expect only features common to the two models to be responsible for the metastability and other non-trivial properties in the large system limit.

We can now consider common features in the distributions. In so far as a marginalised distribution might provide insight, it appears fairly clear that there is a competition between a mean dominated field producing good reconstruction and a variance dominated field leading to only marginal bias in favour of correct reconstruction. The field presumably projects into one of a number of local minima. When $\mathcal{Q}$ is small the variance dominates and there is a weak net alignment with $\vec{b}$. As one increases $\mathcal{Q}$ the mean grows more quickly than the spread, so that in the large $\mathcal{Q}$ limit the state is very orderly. By contrast as one increases $\alpha$ the mean is suppressed by comparison with the spread in the field (and in the couplings), so that one might expect the state to be variance dominated.

The couplings are very different in the modulated models (sparse and dense) by comparison the unmodulated model. In the modulated model one has a random coupling, which one might expect would induce behaviour comparable to a random spin glass or the Hopfield model [MPV87], with a highly nontrivial distribution of local solutions (when ignoring the field). However, by investigation of the unmodulated model we see the space determined entirely by the couplings is a trivial one, corresponding to the sparse ferromagnet and uncorrelated in magnitude (or topology for the regular ensemble) to the fields. We must however consider the strong couplings between fields on neighbouring sites - if we consider these as uncoupled then the model becomes simpler, gauging the dynamic variables to the orientation $\vec{b}$, we have a sparse Mattis model [MPV87] in a random field. Although plausable we have not been able to establish if such a model is capable of the metastability of the type investigated; alternatively, the couplings between fields might form an interesting percolating process responsible for long range order, hence being very different from a Mattis model. An understanding at this level might form the basis for principled global search methods guaranteed to avoid metastable regimes, which might then be generalised to the coupled models.

\section{CONCLUSION}

A comparison of the marginal distributions in the two sparse cases indicates a substantial difference in the coupling. This adds credence to the notion that it is a competition in the field terms which is primarily responsible for the emergence of metastability (as might have been guessed from only the modulated models). In one case the couplings are similar to those of a sparse spin glass, in the other the couplings are uniform. For the unmodulated code we might hope to make use of the fact that we know the equilibrium state of such a model is attracted to a ferromagnetic state, due to the uniform coupling $\mathbf{s}$. By suppressing the emergence of these two states in local search algorithms, either by static factors or dynamical global constraints during the local search, the algorithm may well be improved. Whether or not the high bit error rate metastable state is sufficiently correlated with one of the two ferromagnetic states, and appreciably more so than the low bit error rate solution, would determine whether this may be enough to escape metastable suboptimal solutions. Certainly long range correlations must play a role in the dynamical relaxation process and the simplicity of the couplings in the unmodulated system ought to offer some insight. It would also be interesting to see what similarities remain between the modulated and unmodulated sparse codes in a wider range of optimal detectors (varying $\beta$ ) and with respect to heuristic methods.

\section{ACKNOWLEDGMENT}

Support from EVERGROW, IP No. 1935 in FP6 of the EU and EPSRC grant EP/E049516/1 are gratefully acknowledged.

\section{REFERENCES}

[GW07] D. Guo and C. Wang. Multiuser detection of sparsely spread cdma. (unpublished), 2007.

[Kab03] Y. Kabashima. A cdma multiuser detection algorithm on the basis of belief propagation. Jour. Phys. A, 36(43):11111-11121, 2003.

[MMU05] C. Measson, A. Montanari and R. Urbanke. Maxwell Construction: The Hidden Bridge between Iterative and Maximum a Posteriori Decoding. Preprint arXiv:cs/0506083, 2005.

[MPT06] A. Montanari, B. Prabhakar, and D. Tse. Belief propagation based multiuser detection. In Proceedings of the Allerton Conference on Communication, Control and Computing, Monticello, USA, 2006.

[MPV87] M. Mezard, G. Parisi, and M.A Virasoro. Spin Glass Theory and Beyond. World Scientific, 1987.

[RS07] J. Raymond and D. Saad. Sparsely spread CDMA - a statistical mechanics-based analysis Jour. Phys. A, 40(41),12315-12334,2007.

[Tan02] T. Tanaka. A statistical-mechanics approach to large-system analysis of cdma multiuser detectors. Information Theory, IEEE Transactions on, 48(11):2888-2910, Nov 2002.

[Ver98] S. Verdu. Multiuser Detection. Cambridge University Press, New York, NY, USA, 1998.

[YT06] M. Yoshida and T. Tanaka. Analysis of sparsely-spread cdma via statistical mechanics. In Proceedings - IEEE International Symposium on Information Theory, 2006., pages 2378-2382, 2006. 